\title{
Multidisciplinary approach for improving aesthetics in a cleft lip and palate patient
}

\author{
Khalid Al Zoman ${ }^{1, *}$, Christer Henningsson ${ }^{2}$, Atheer Binzouman ${ }^{3}$, Sultan Al Mubarak ${ }^{4}$, Tariq Al Ali ${ }^{5}$ \\ ${ }^{1,4}$ Consultant Periodontist, ${ }^{2}$ Associate Professor, ${ }^{3}$ Resident, ${ }^{5}$ Consultant Restorative Dentist, Dept. of Dental, ${ }^{1,3-5}$ King Faisal Specialist \\ Hospital, Riyadh, Saudi Arabia, ${ }^{2}$ Gothenburg University, Sweden
}

*Corresponding Author: Khalid Al Zoman

Email: zoman@kfshrc.edu.sa

\begin{abstract}
A 27-year-old woman with a right-sided cleft lip and palate and a left sided cleft lip and congenitally missing teeth \#s12 and 11. After primary treatment of the cleft during childhood and adolescence, the teeth were still missing and a large defect in the alveolar crest remained. Patient's chief complaint was poor aesthetics in the upper anterior region. This case presentation describes how, with a multidiscipline approach by means of orthodontics, soft tissue grafting and a tooth-supported fixed partial denture the conditions were treated to achieve as good aesthetical result as possible.
\end{abstract}

Keywords: Cleft Lip and palate, Ridge augmentation, Multidiscipline approach.

\section{Case Report}

A 27 -year old female patient with a chief complaint related to the appearance of her maxillary anterior dentition. The patient was dissatisfied with the gingival aesthetics as wells as the poor functioning removable appliance. Evaluation of the patient's history revealed that the patient had undergone surgical correction of a bilateral cleft lip and unilateral cleft palate as a child and for the last 15 years had worn a removable partial denture to replace teeth \#'s12 \& 11. Her medical history was noncontributory and the patient did not smoke. Extra-oral examination indicated slight asymmetry in the upper lip, straight profile, and exposure of gingival cleft while smiling. Intra-oral examination revealed a sagittally neutral bite with posterior crossbite. Teeth \# 14, 12, 11 and \# 24 were congenitally missing. (Fig.1 a,b). Periodontal examination revealed relatively healthy dentition with moderate plaque accumulation, no probing greater than $3 \mathrm{~mm}$, and fair oral hygiene. Localized slight gingival inflammation and bleeding were noted on upper anterior teeth.
At the site of the cleft, the residual ridge defect was classified as a Siebert Class III (deficiency in a buccopalatal as well as apicocoronal direction) with a through-andthrough residual cleft defect. ${ }^{1}$ Radiographic examination revealed a radiolucent "suture-like" area and a vertical osseous defect within the cleft; adjacent bone levels appeared normal.

As developed by the various members of the restorative team, three restorative options were presented to the patient:

1. Orthodontic alignment and a removable partial denture.

2. Orthodontic alignment, soft tissue augmentation, and tooth supported bridge with extension from tooth \#13\#24.

3. Orthodontic alignment, bone and soft tissue augmentation and implant supported bridge and crown in area of teeth \#12-\#11 and \#24.

For this patient, it was assessed that option II would achieve most of the objectives.

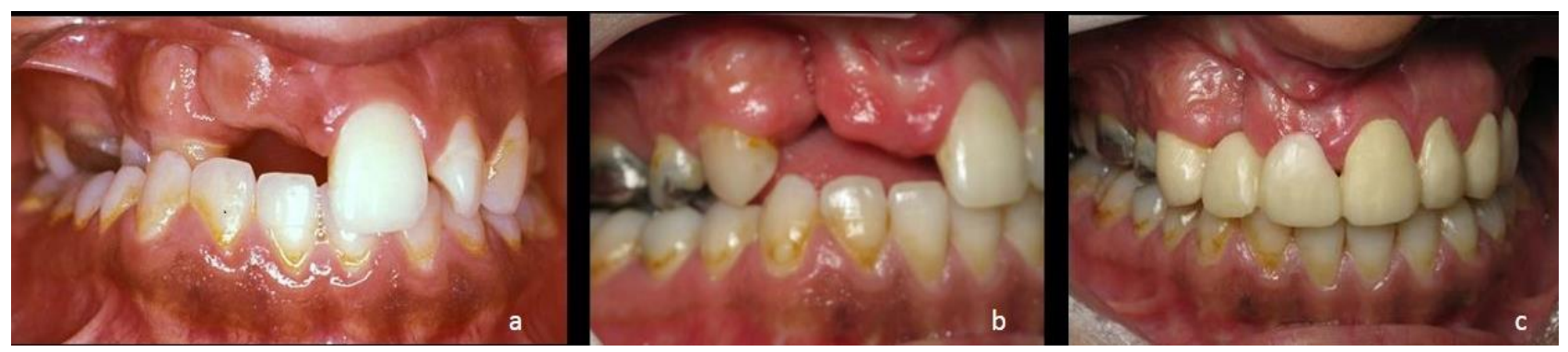

Fig. 1: Pre-Orthodontic treatment a): Frontal view of class III ridge defect with residual cleft palate and posterior cross bite; b): Post-orthodontic treatment. Right lateral view of class III ridge defect with residual cleft palate; c): Provisional bridge in place, note the marked concavity buccal of tooth \#2

\section{Orthodontic Treatment}

The primary objective of orthodontic therapy was to level and align the anterior maxillary and mandibular segments and to correct the right posterior crossbite. Establish proper pontic space, and shift the patient's midline. This was crucial for achieving a satisfactory aesthetic result. The maxillary arch was expanded, and the axial inclinations of the anterior teeth (both buccolingual and mesiodistal) were corrected (Fig. 1b, c). The treatment did not include stripping the teeth. The orthodontic treatment required approximately 12 months of fixed 
appliance therapy. The patient wore a removable appliance to replace tooth \# 12\&11 during the orthodontic treatment.

\section{Periodontal Treatment}

The residual ridge defect was classified as a Siebert Class III with a through-and-through residual palatal cleft. It was therefore determined that by utilizing an onlay graft technique in combination with the subepithelial connective tissue graft, defect closure could be accomplished. Trapezoidal incision extending from palatal tissue of the cleft defect and including the buccal cleft tissue was carried out (Fig. 2 a,b). The trapezoidal partial-thickness flap was excised and the epithelial tissue was removed from the cleft area. Thick onlay gingival graft was harvested from the crestal and palatal tissue of the missing tooth \# 15. A small piece of connective tissue was dissected from the onlay graft and was utilized to close the cleft palate (Fig. $2 \mathrm{c}$ ). The onlay gingival graft was used to close the cleft defect as well as to gain apicocoronal and buccolingual ridge augmentation (Fig.2 d). The graft was stabilized with 5-0 Vicryl sutures. The apical aspects of the pontics of the interim prosthesis were reduced to prevent the surgical site from creating pressure against the pontics as a result of the likely swelling of the augmented area.

Post-operative recommendations included chlorhexidine $(0.2 \%)$ rinsing for one minute, twice a day, use of prescribed analgesics (Brufen $600 \mathrm{mg}$ ) as needed for severe pain, application of cold compresses for 20 minutes every two hours the first day after the operation, and restriction of sports or physical activity for up to ten days after the operation.

The patient presented 10 weeks post-surgery with complete defect closure and significant improvement in ridge form. A minor defect was still present resulting in the need for additional augmentation (Fig. 2 e). A combined onlay-interpositional gingival graft was harvested to slightly augment the crestal and buccal tissue area of tooth \# 12. The recipient bed was created via the de-epithelization of the coronal portion of the edentulous ridge and via preparation of a split thickness flap buccal of area of tooth \# 12 with releasing incisions (Fig. 3 a). The graft was stabilized with 5-0 Vicryl sutures (Fig.3 b,c). A significant improvement in ridge form was evident following 12 weeks of healing (Fig. $4 \mathrm{a}, \mathrm{b})$

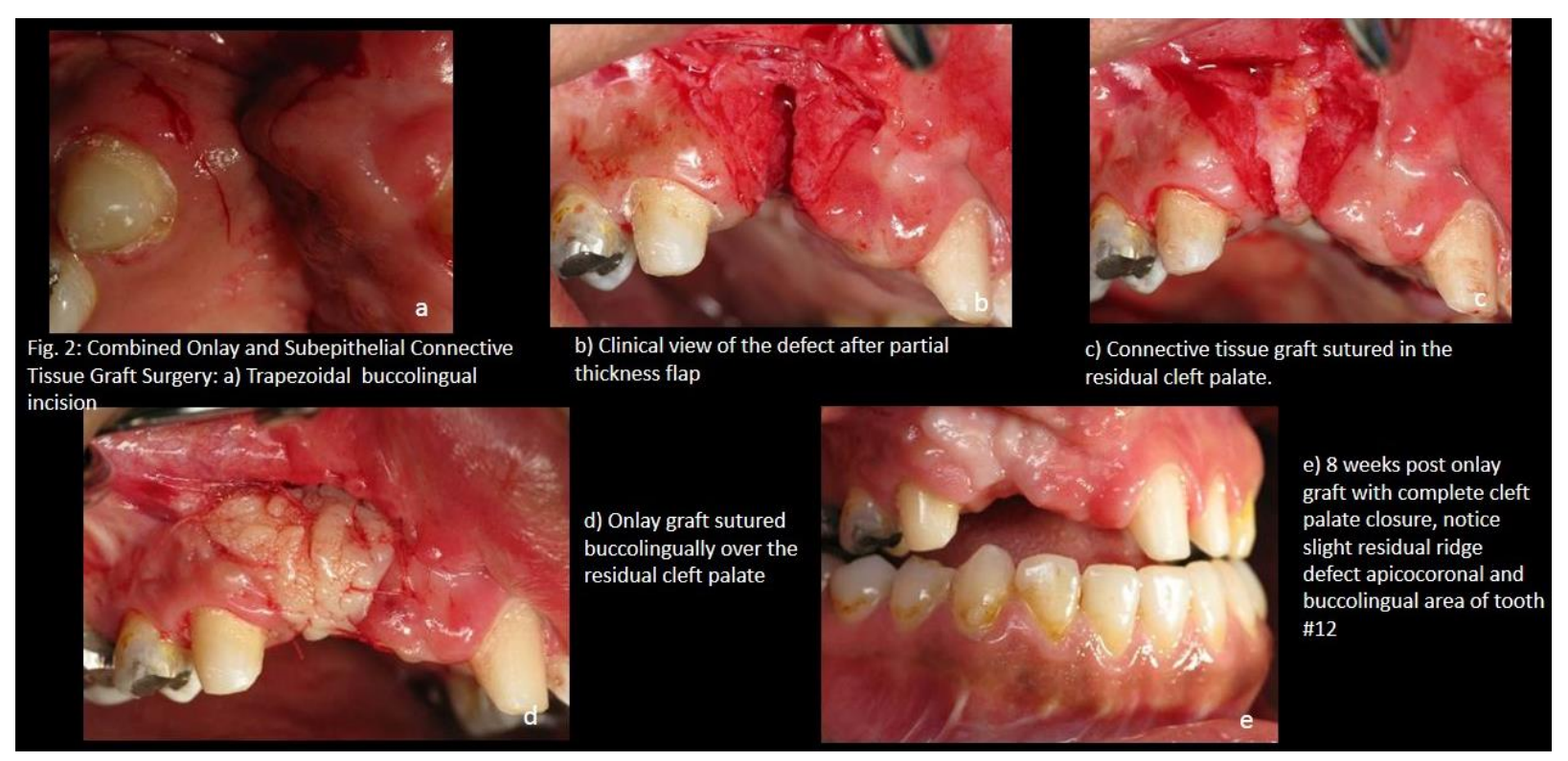



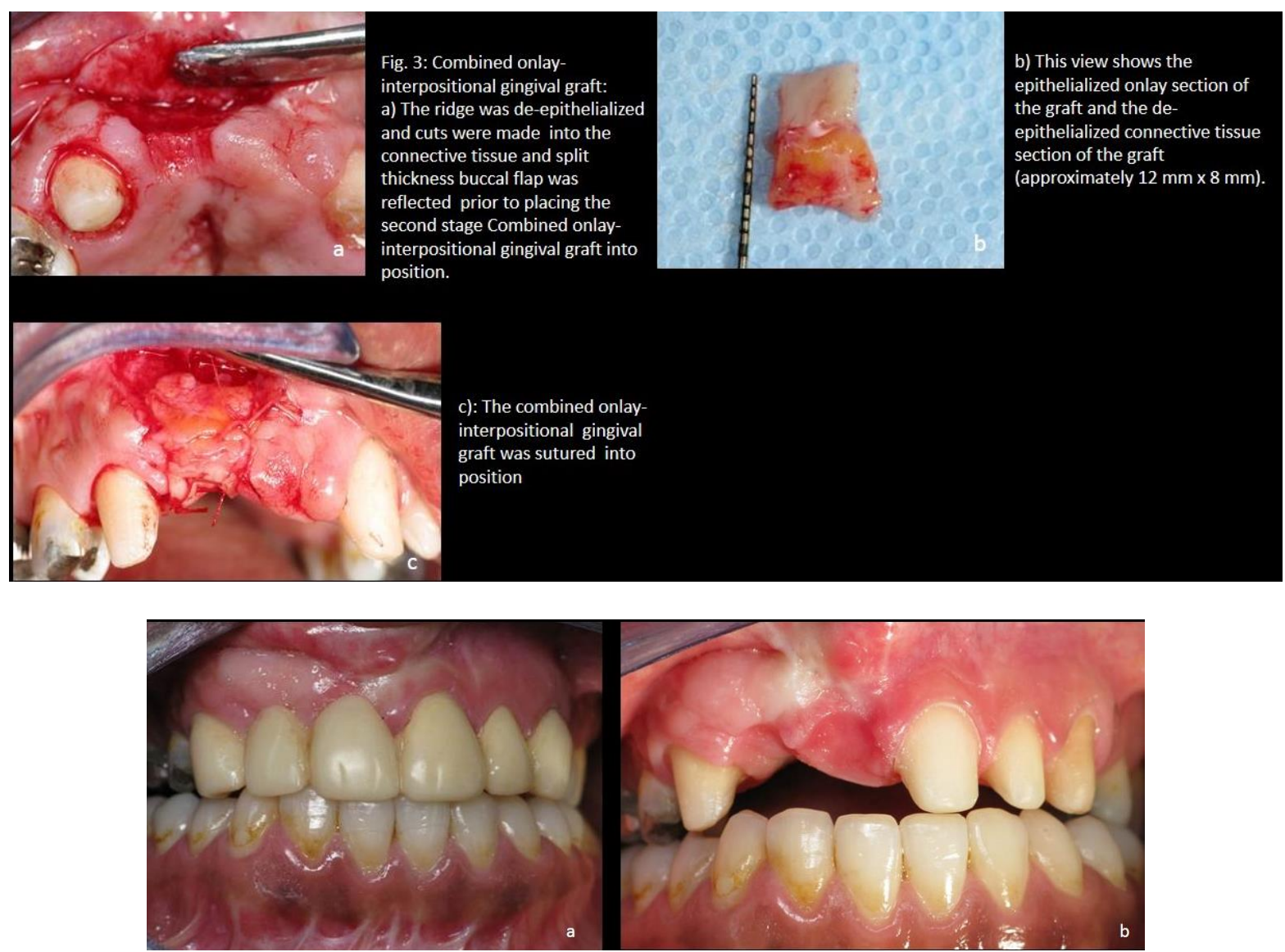

Fig. 4: Three months post-surgery. a): Marked increase in tissue volume. Complete palatal defect closure and good interdental papilla height between \#11 and \#12; b): Note good interdental papilla height between area of teeth \#11 and $\# 12$
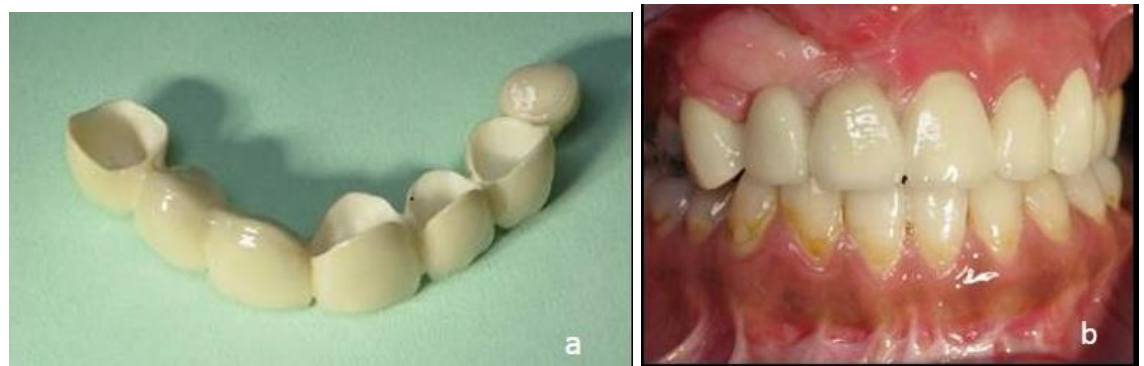

Fig. 5: Cementation of the ceramic bridge: a): The pontics were designed with a sharp edge cervically and buccally; b): 2 months post-cementation. Marked increase in tissue volume. Complete palatal defect closure and good interdental papilla height between teeth \#11 and \#12

\section{Prosthetic Treatment}

After orthodontic treatment but before soft tissue grafting the teeth \#s13,21,22,23 were prepared and was fitted with a temporary bridge (Fig. 1 c) in heat cured Polymethylmethacrylat, Coldpack Tooth Acrylic (The Motloid Company, Chicago, Ill, USA) cemented with Temp Bond NE temporary cement (KERR Corporation, Orange, CA,USA), In order not to interfere with the soft tissue during treatment and in order to get good dimension of the temporary bridge the preparations were made with a rounded shoulder that was placed slightly above the gingival margin. No cantilever pontic at tooth \#24 was present in the temporary bridge. At the same visit, the teeth \#42 \& \#43 was shortened and re-contoured by grinding.

Final preparations were made with Procera $\AA$ Preparation Kit, (Nobel Biocare, Zürich, Switzerland). Preparations were finished to a chamfer design, Ultrapac retraction cords (Ultradent products Inc, South Jordan, UT, USA) were used without any astringents or vasoconstrictors were used. Impressions were made with Imprint (3M/ESPE, St Paul, MN, USA) and a custom tray. Bite relation was recorded with Blue Mousse (Parkell Inc, Edgewood, NY, USA).

The framework was made of zirconium dioxide and Cad Cam technique using the Procera ${ }^{\circledR F}$ Forte scanner (Nobel 
Biocare, Zürich, Switzerland). The copings were $0.7 \mathrm{~mm}$ thick and the connectors and pontics were designed according to the manufacturer's recommendations. The Framework was scanned and designed (CAD) at the Dental Laboratory at King Faisal Specialist Hospital \& Research Center in Riyadh, Kingdom of Saudi Arabia and the file was linked to the Procera ${ }^{\circledR}$ production plant in Vastberga, Sweden, where the framework was manufactured (CAM).

After try-in the bridge was cemented with glassionomer cement, KetacCem ((3M/ESPE, St Paul, MN, USA)). The pontics were designed with a sharp edge cervically and buccally and slightly overextended so the pontics caused a slight compression of the soft tissue at cementation (Fig. 5 $\mathrm{a}, \mathrm{b})$.

The esthetics and the functional ceramic were made at the Dental Laboratory at King Faisal Specialist Hospital \& Research Center in Riyadh.

\section{Discussion}

The most common causes of alveolar ridge deformities include developmental defects, advanced periodontal disease, traumatic removal of teeth, and surgical injury. ${ }^{2,3}$ The reconstructive periodontal procedure permits the restoration of the hard and soft tissues of the alveolar ridge to their former dimensions and gives the restorative dentist the opportunity to provide their patients with a functional and improved esthetic prosthesis., ${ }^{4,5}$

Treatment of patients with cleft lip and palate calls for a complex multidisciplinary approach with long-term involvement. The team concept remains the key to success in the care of these patients. Periodontists are integral members of this team. Plastic surgeons, orthodontists, and prosthodontists are part of the cleft palate team responsible for the medical care that, in many patients, starts shortly after birth and continues in various stages until maturity. ${ }^{6}$

Resorbed ridges may be classified as Class I, II or III ${ }^{1}$. A Class I ridge has predominantly horizontal loss of dimension. This is the most often seen when one tooth is lost, especially if the facial plate of bone was damaged. A Class II ridge deformity involves loss of height of the ridge. The Class III defect has both horizontal and vertical loss, often seen when adjacent teeth are missing. ${ }^{1}$

In many cases, these defects may be repaired by utilizing the patient's own palatal connective tissue. Class I defects may be repaired with the submucosal connective tissue graft (pouch procedure). ${ }^{7}$

Class II and Class III defects may require an alternative approach because of the greater amount of ridge to be restored. Subepithelial connective tissue grafts, ${ }^{8}$ onlay grafts, ${ }^{1}$ and interpositional onlay ${ }^{9}$ grafts, have been widely used for the correction of Class II and Class III ridge deformities.

The onlay connective tissue graft is basically a large free gingival graft placed in top of the ridge in order to increase both ridge height and width. ${ }^{1}$ As with the submucosal graft, the donor tissue is harvested from the palate. In this procedure, however, the epithelial surface remains attached to the connective tissue. The graft must be intimately sutured to the bleeding recipient site that has been prepared. As the graft survival initially depends on plasmatic circulation, absolute stability of the graft is essential (Fig. $2 \& 3$ ).

Another procedure useful for correcting small Class II and Class III defects is the combined onlay-interpositional gingival graft. This procedure incorporates the best features of the onlay graft with the best features of the interpositional and subepithelial connective tissue graft procedures. ${ }^{9}$ In this way, ridge height as well as width may be enhanced by a single procedure.

The ridge defect in this patient was classified as a Class III. A combined onlay-interpositional gingival graft was selected to eliminate the soft tissue defect and give adequate buccolingual and apicocoronal width needed for the placement of a functional and esthetic pontic. In addition to eliminating the soft tissue ridge defect, this surgical procedure also created a convex-shaped ridge where the ovate pontic would look as if it emerged from the ridge. The interproximal papillae filled the embrasure space between the pontic and teeth.

Because of the extent of the ridge defect, performing more than one procedure may be necessary to achieve the desired result. An onlay graft may precede a combined onlay-interpositional gingival graft, or vice versa. As part of the initial treatment planning and consultation process, the patient must be made aware of the potential for multiple procedures. The patient must be prepared for the additional psychological expense, as well as the time, postoperative discomfort, and financial commitments this type of therapy demands. Rarely, especially in severe cases, will the desired clinical outcome be achieved with one operation.

When prosthetically replacing the missing teeth \#12, \#11 and \#24 three options were available as previously mentioned.

Alternative I, the removable partial denture, was excluded because of its low degree of rehabilitation and since the patient had had one previously and was not satisfied with it.

Regarding alternative III, it was assessed that available bone volume in area of teeth \#12 and \#11 was insufficient for placing implants. In order to host dental implants, extensive augmentation with bone and soft tissue would be necessary since the bone bridge between the maxillary segments at the cleft was very delicate and narrow. In order to be able to cover the graft with soft tissue, it would have to be made in several surgical sessions. The scar formation due to previous surgical interventions made it hard to predict the result of this kind of procedure. It is also hard to create good esthetic results in the soft tissue around multiple implants. This is especially sensitive in the esthetic zone. If implants were to be placed, tooth \#25 would have to be orthodontically straightened and repositioned. This would mean more extended orthodontic treatment. With this alternative, the esthetics of the patient's natural teeth in the anterior could not be changed. Since the patient was not satisfied with the appearance of her natural teeth crowns would still have to be made. 


\section{Conclusion}

The case presented in this article shows that with a multidiscipline approach good aesthetic results can be achieved even though the conditions at the start are poor. A thorough and comprehensive treatment plan is crucial to the outcome of the treatment and it has to include all parties involved in the treatment. Since this is a case report, no extensive conclusions can be drawn from it but it describes the possibility to improve the patient's aesthetical and functional situation even though the conditions at the start are severely compromised. It also demonstrates the importance of restoring the soft tissue at the site of the defect with viable soft tissue instead of a prosthetic material to achieve an optimal result. The case also indicates the need for more systematical research in this area to predict results of the treatment and to facilitate the treatment planning for the care provider.

\section{Conflict of Interest: Nil}

\section{References}

1. Seibert JS. Reconstruction of deformed partially edentulous ridges, using full thickness onlay grafts. Part I. Technique and wound healing. Compend Contin Educ Dent. 1983;4:437-453.

2. Allen EA, Gainza CS, Farthing GG. DA. Improved technique for localized ridge augmentation. J Periodontol. 1985;56:195199.

3. Morley J. The role of cosmetic dentistry in restoring a youthful appearance. J Am Dent Assoc. 1999:130:1166-1172.

4. Hurzeler MB, Weng D. Functional and esthetic outcome enhancement of periodontal surgery by application of plastic surgery principles. Int J Periodontics Restorative Dent. 1999:19:36-43.

5. Seibert JS. Ridge augmentation to enhance esthetics in fixed prosthetic treatment. Comp Contin Dent Educ Dent. 1993; 12:548-561.

6. Saunders ID, Geary L, Fleming P, Gregg TA. A simplified feeding appliance for the infant with a cleft lip and palate. Quintessence Int. 1989;20:907-910.

7. Abrams H, Kopczyk R, Kaplan AL. Incidence of anterior ridge deformities in partially edentulous patients. J Prosthet Dent. 1987;57:191-194.

8. Langer B. Calagna $\mathrm{L}$ The subepithelial connective tissue graft. J Prosthet Dent. 1980;44:363-367.

9. Seibert JS, Louis JV (1996) Soft tissue ridge augmentation utilizing a combination onlay-interpositional graft procedure: a case report. Int J Periodontics Restorative Dent. 1996;16(4):310-321.

10. Benington, I. C., Watson, I. B., Jenkins, W. M. M., and Allan, G. R. J.: Restorative Treatment of the Cleft Palate Patient. $\mathrm{Br}$ Dent. 1979;146(3):79-82.

How to cite this article: Zoman K. A, Henningsson C, Binzouman A, Mubarak S. A, Ali T A. Multidisciplinary approach for improving aesthetics in a cleft lip and palate patient. Int J Oral Health Dent. 2018;4(4):234-238. 